\title{
The diagnostic value of dopamine transporter imaging and olfactory testing in patients with parkinsonian syndromes
}

Charalampos Georgiopoulos, Anette Davidsson, Maria Engström, Elna-Marie Larsson, Helene Zachrisson and Nil Dizdar

\section{Linköping University Post Print}

\section{Tweet}

N.B.: When citing this work, cite the original article.

The original publication is available at www.springerlink.com:

Charalampos Georgiopoulos, Anette Davidsson, Maria Engström, Elna-Marie Larsson, Helene Zachrisson and Nil Dizdar, The diagnostic value of dopamine transporter imaging and olfactory testing in patients with parkinsonian syndromes, 2015, Journal of Neurology, (262), 9, 21542163.

http://dx.doi.org/10.1007/s00415-015-7830-4

Copyright: Springer Verlag (Germany)

http://www.springerlink.com/?MUD=MP

Postprint available at: Linköping University Electronic Press

http://urn.kb.se/resolve?urn=urn:nbn:se:liu:diva-122669 


\section{The diagnostic value of dopamine transporter imaging and olfactory testing in patients with parkinsonian syndromes}

Charalampos Georgiopoulos 1,6

Anette Davidsson ${ }^{2}$

Maria Engström ${ }^{3,6}$

Elna-Marie Larsson ${ }^{4}$

Helene Zachrisson ${ }^{2, \#}$

Nil Dizdar ${ }^{5, \#}$

${ }^{1}$ Department of Radiology and Department of Medical and Health Sciences, Linköping University, 58185 Linköping, Sweden

${ }^{2}$ Department of Clinical Physiology and Department of Medical and Health Sciences, Linköping University, 581 85 Linköping, Sweden

${ }^{3}$ Department of Medical and Health Sciences, Linköping University, 58185 Linköping, Sweden

${ }^{4}$ Department of Surgical Sciences/Radiology, Uppsala University, Akademiska sjukhuset, 75185 Uppsala, Sweden

${ }^{5}$ Department of Neurology and Department of Clinical and Experimental Medicine, Linköping University, 581 85 Linköping, Sweden

${ }^{6}$ Center for Medical Image Science and Visualization (CMIV), Linköping University, 58185 Linköping, Sweden

${ }^{\#}$ Helene Zachrisson and Nil Dizdar have contributed equal parts for last authorship

\section{Corresponding author:}

Charalampos Georgiopoulos

Tel: +46101032700

Email: Charalampos.Georgiopoulos@regionostergotland.se 


\section{ABSTRACT}

Purpose: To compare the efficacy of olfactory testing and presynaptic dopamine imaging in diagnosing Parkinson's disease (PD) and Atypical Parkinsonian Syndromes (APS); to evaluate if the combination of these two diagnostic tools can improve their diagnostic value.

Methods: A prospective investigation of 24 PD patients, 16 APS patients and 15 patients with non-parkinsonian syndromes was performed during an 18-month period. Single Photon Emission Computed Tomography with the presynaptic radioligand ${ }^{123}$ I-FP-CIT $\left(\right.$ DaTSCAN $\left.^{\circledR}\right)$ and olfactory testing with the Brief 12-item Smell Identification Test (B-SIT) were performed in all patients. DaTSCAN was analysed semi-quantitatively, by calculating two different striatal uptake ratios, and visually according to a predefined ranking scale.

Results: B-SIT score was significantly lower for PD patients, but not significantly different between APS and Non-parkinsonism. The visual assessment of DaTSCAN had higher sensitivity, specificity and diagnostic accuracy compared to olfactory testing. Most PD patients (75\%) had visually predominant dopamine depletion in putamen, while most APS patients (56\%) had visually severe dopamine depletion both in putamen and in caudate nucleus. The combination of DaTSCAN and B-SIT led to a higher rate of correctly classified patients.

Conclusions: Olfactory testing can distinguish PD from Non-parkinsonism, but not PD from APS or APS from Non-parkinsonism. DaTSCAN is more efficient than olfactory testing and can be valuable in differentiating PD from APS. However, combining olfactory testing and DaTSCAN imaging has a higher predictive value than these two methods separately.

Keywords: Parkinson's disease, Atypical Parkinsonism, Parkinsonian syndromes, olfaction, ${ }^{123}$ I-FP-CIT SPECT 


\section{INTRODUCTION}

The clinical diagnosis of Parkinson's disease (PD) is currently based on the presence of cardinal motor features and positive response to dopaminergic therapy [1]. However, it can be difficult to differentiate PD from other parkinsonian syndromes with similar signs and symptoms, especially in the early stages of the disease. Diseases that can imitate the clinical image of PD are grouped as Atypical Parkinsonian Syndromes (APS) or Secondary Parkinsonism (SP) [2] . Despite the similar clinical image of PD and other parkinsonian syndromes in the early stages, prognosis differs significantly [3]. Therefore, a reliable method that can differentiate PD from other parkinsonian syndromes is essential.

In recent years, single photon emission computed tomography (SPECT) with the presynaptic radiotracer ioflupane, also known as DaTSCAN ${ }^{\circledR}$, has been widely used as a diagnostic test to detect loss of functional dopaminergic neuron terminals in striatum [4]. DaTSCAN imaging has high diagnostic performance in separating patients with PD from patients with non-parkinsonian syndromes $[5,6]$. However, the efficacy of DaTSCAN imaging in separating PD from APS has been a matter of controversy [4, 7-10].

Olfactory dysfunction in patients with PD has been confirmed since several years; it occurs in the early stages of the disease and is independent of treatment or age at onset [11-16]. Olfactory function can vary from normal to strongly impaired in APS [17-19]. Olfactory testing is officially recommended by the European Federation of Neurological Societies and the Movement Disorder Society in order to differentiate PD from other parkinsonian disorders [20].

The main objective of this study was to compare DaTSCAN imaging and simple olfactory testing in terms of accuracy and efficacy in diagnosing parkinsonian syndromes. We analysed DaTSCAN SPECT with two different approaches: semi-quantification and visual assessment of DaTSCAN uptake. Moreover, we wanted to assess the diagnostic contribution of the combined results from DaTSCAN imaging and olfactory testing in separating PD from APS.

\section{MATERIALS AND METHODS}

This study was conducted in accordance with the current revision of the Declaration of Helsinki and was approved by the Regional Ethical Review Board in Linköping, Sweden (registration number 2011/415-31). 


\section{Patients}

All patients who were referred to the Department of Nuclear Medicine for DaTSCAN SPECT, during an 18month period, were asked for inclusion in the study. Patients were referred and followed-up by neurologists and geriatricians from the Southeast region of Sweden. Written consent was obtained by all patients who agreed to participate. Co-morbid medical conditions, such as severe head injury, intracranial surgery, surgery in the nasal cavity, seasonal allergies, sinusitis or other current respiratory infection, as well as current smoking served as exclusion criteria.

The following diagnosis groups were defined in this study:

i. PD: patients diagnosed with idiopathic Parkinson's Disease

ii. APS: patients with Multiple System Atrophy (MSA), Progressive Supranuclear Palsy (PSP), Dementia with Lewy Bodies (DLB) or unspecified APS syndrome

iii. Secondary Parkinsonism (SP): patients with vascular parkinsonism or drug-induced parkinsonism

iv. Non-parkinsonism: patients with essential tremor, postural tremor or other pathology that was not related to the basal ganglia.

\section{Olfactory Evaluation}

In order to simulate clinical practice, where time-efficiency matters, we chose to use the Brief 12-item Smell Identification Test (B-SIT, Sensonics, Inc., New Jersey, USA), among the various available testing paradigms. This test consists of a booklet with 12 different microencapsulated odorants. In each page, there is a multiplechoice question with four alternative responses and one odorant embedded in a microcapsule at the bottom of the page. Each odorant is released by scratching the microcapsule, and the patient is then required to choose the alternative that corresponds to the odorant. The performance of each patient is presented as B-SIT score, which has a range from 0 to 12 .

An examiner, blind to participants' clinical status, explained the test beforehand and helped all patients by scratching the booklets and marking their answers. We used the norms provided by the manufacturer in order to classify the patients as normosmic, hyposmic or anosmic in accordance to their sex and age. Prior to the BSIT examination, all participants were asked to self-evaluate their olfactory ability as impaired or normal.

\section{$\operatorname{DaTSCAN}^{\circledR}$}


The iodine-labelled ligand ioflupane $\left({ }^{123} \mathrm{I}-\mathrm{FP}-\mathrm{CIT}\right)$ was employed for DaTSCAN SPECT (GE Healthcare, Eindhoven, the Netherlands). Ioflupane is a presynaptic radioligand with affinity to the presynaptic dopamine transporter protein, in the striatal dopaminergic terminals. Image acquisition, processing and automated semiquantitative evaluation were performed as previously described by our group [7]. We used EXINI DAT (EXINI Diagnostics AB, Lund, Sweden) for the quantification of our data. EXINI DAT is CE-approved software, which allows automated, supervised quantification of DaTSCAN SPECT, including partial volume effect correction. The specific ligand binding to putamen and caudate nucleus was calculated, with the occipital lobe serving as reference for non-specific binding. Two ratios were calculated: the ratio between the uptake in striatum and that in the occipital lobe, hereinafter mentioned as striatum ratio, and the ratio between the uptake in putamen and that in caudate nucleus, hereinafter mentioned as putamen/caudate ratio.

An experienced nuclear medicine technician and an experienced physician, both blinded to patients' clinical status, performed individually visual assessment of all transaxial SPECT images. The uptake pattern of the ligand was classified in five different stages, as previously proposed by Kahraman et al. [8] and as shown in Figure 1. These five stages are listed below:

1. Burst striatum: severe bilateral reduction, with almost no uptake in putamen or caudate nucleus.

2. Egg shape: bilateral uptake reduction in both putamina and normal or borderline normal uptake from caudate nucleus, creating an oval shape.

3. Mixed type: asymmetrical ioflupane uptake, with reduced uptake in the putamen of one side.

4. Eagle wing: borderline normal, symmetrical ioflupane uptake, with only discrete reduction in one or both putamina, creating the shape of a wing.

5. Normal: symmetrical ioflupane uptake in putamen and caudate nucleus bilaterally.

\section{Statistical analysis}

Kruskal-Wallis one-way ANOVA was employed to investigate potential differences in age and illness duration among groups. Fisher's exact test was used to investigate potential differences in sex among groups. Pearson's Chi-square test was used to compare the results of visual assessment of DaTSCAN. In order to identify potential differences in the B-SIT score and the semi-quantitative analysis, we used non-parametric Kruskal-Wallis oneway ANOVA, with Mann-Whitney U Test as post hoc test for pairwise comparisons; for significant differences, the p-value from Mann-Whitney U Test is presented. The analysis of the B-SIT score was cross-examined for possible confounders with ANCOVA, by using age as covariate and sex as additional factor. Correlation was 
assessed by Pearson coefficient (r). Sensitivity, specificity, positive predictive value, negative predictive value and accuracy were calculated for the B-SIT score and the visual evaluation. The semi-quantitative analysis of DaTSCAN produces two striatal uptake ratios for each patient, one for each hemisphere of the brain; the lower of these two ratios was used in the statistical analysis. The results of semi-quantification depend on the type of camera, collimator, acquisition and reconstruction parameters, and are, therefore, not easily comparable within different SPECT systems [21]. Hence, we did not to calculate the sensitivity, specificity, positive predictive value and negative predictive value for the semi-quantitative analysis of DaTSCAN. Binary logistic regression was employed in order to estimate the predictive value of B-SIT and of the visual assessment of DaTSCAN. Statistical analysis was performed using IBM $^{\circledR}$ SPSS $^{\circledR}$ Statistics v22. For all tests, $\mathrm{p}<0.05$ was considered statistically significant. All results are presented in the form median value with lower and upper 95\% confidence intervals (CI). The abbreviation df will be hereinafter used to state the degrees of freedom.

\section{RESULTS}

\section{Patients}

In total 67 patients were recruited in the study. One patient was excluded from data analysis due to previous intracranial surgery and another patient was excluded because of previous surgery in the nasal cavity. One patient died of cancer before receiving a diagnosis regarding his parkinsonism, and he was, therefore, excluded. One patient could not complete the DaTSCAN examination due to claustrophobia and was also excluded. Three patients had to be excluded due to uncertain diagnosis several months after recruitment. The remaining 60 patients were divided into four groups, according to their diagnosis: PD (24 patients), APS (16 patients), SP (5 patients), Non-parkinsonism (15 patients). Among the patients with APS, 6 were diagnosed with MSA, 4 with PSP, 1 with DLB and 5 with unspecified form of APS. Among the patients with SP, 3 were diagnosed with vascular parkinsonism and 2 with drug-induced parkinsonism. The SP group was too small to be included in the statistical comparisons among groups; it was however included in the comparisons between parkinsonism (all PD, APS and SP patients) and Non-parkinsonism. The demographics of each group, including age, sex, illness duration and medication at the time of the study are presented in Table 1. Kruskal-Wallis one-way ANOVA showed no significant difference regarding age and illness duration among groups. Fisher's exact test showed no significant difference regarding sex among groups.

\section{Olfactory self-evaluation}


In the PD group $50 \%$ of the patients were aware of some smell impairment vs. $38 \%$ in the APS group and $20 \%$ in the Non-parkinsonism group. Pearson's Chi-square showed no significant difference between groups. There was a weak positive correlation between smell self-evaluation and B-SIT score in PD patients (Pearson correlation $\mathrm{r}=0.458, \mathrm{p}=0.024$ ), but no significant correlation in the APS or Non-parkinsonism group.

\section{Olfactory evaluation with B-SIT}

The median value of B-SIT score in the PD group was 4.0 (95\% CI: 3 and 4.5) vs. 6.0 (95\% CI: 4 and 9) in the APS group and 8.0 (95\% CI: 7 and 9) in the Non-parkinsonism group. Kruskal-Wallis one-way ANOVA, with Mann-Whitney U test as post hoc test, showed significant difference between PD and Non-parkinsonism ( $\mathrm{p}<0.001,3 \mathrm{df}$ ), but no significant difference between PD and APS or APS and Non-parkinsonism. After using age as a covariate and sex as an additional factor (ANCOVA), there was still significant difference between PD and Non-parkinsonism ( $\mathrm{p}<0.001,3 \mathrm{df}$ ). There was no significant correlation between B-SIT score and illness duration in PD, APS or Non-parkinsonism. Based on the B-SIT norms provided with the test, $71 \%$ of PD patients were anosmic and $21 \%$ were hyposmic vs. $31 \%$ anosmic and $38 \%$ hyposmic APS patients (Table 2). Anosmic patients were compared against the combination of normosmic and hyposmic, with Pearson's Chi-square test; there were significantly more anosmic PD patients than anosmic APS patients ( $\mathrm{p}=0.016,1 \mathrm{df})$.

Patients with impaired olfaction (anosmic and hyposmic) were grouped together and were compared to normosmic patients in terms of sensitivity, specificity, positive predictive value, negative predictive value and diagnostic accuracy for B-SIT. The sensitivity of B-SIT in distinguishing parkinsonism (all PD, APS and SP patients included) from Non-parkinsonism was $80 \%$, the specificity $40 \%$, the positive predictive value $80 \%$, the negative predictive value $40 \%$ and the diagnostic accuracy $70 \%$. By focusing only on PD (PD vs. Nonparkinsonism) the sensitivity was $92 \%$, the specificity $40 \%$, the positive predictive value $71 \%$, the negative predictive value $75 \%$ and the diagnostic accuracy $72 \%$.

\section{$\operatorname{DaTSCAN}^{\circledR}$}

The striatal uptake for all patients was visually categorized in 5 different patterns, as proposed by Kahraman et al. [8] ; the results for each diagnostic group are presented in Table 2. All PD patients had abnormal striatal uptake pattern (Grades 1-3), while one APS patient had normal striatal uptake pattern (Grade 5). In the PD group, $21 \%$ of the patients were classified as Grade 1 "burst striatum" vs. $56 \%$ in the APS group. Moreover, $75 \%$ of PD patients were classified as Grade 2 "egg shape" vs. 19\% for the APS group. Grade 1 was compared against the 
combination of all other grades for PD and APS with Pearson's Chi-square. Similarly, Grade 2 was compared against all other grades. There was significant difference between PD and APS for Grade 1 ( $p=0.025,1 \mathrm{df})$ and Grade 2 ( $\mathrm{p}=0.001,1 \mathrm{df}$ ), as illustrated in Figure 2. The majority (80\%) of Non-parkinsonism patients were classified as normal (Grade 5). However, 13\% were classified as Grade 4 and $7 \%$ as Grade 3.

Patients with abnormal striatal uptake of DaTSCAN (Grades 1-4) were grouped together and were compared to patients with normal striatal uptake (Grade 5) in terms of sensitivity, specificity, positive predictive value, negative predictive value and diagnostic accuracy. The sensitivity of DaTSCAN in distinguishing parkinsonism (all PD, APS and SP patients included) from Non-parkinsonism was $96 \%$, the specificity $80 \%$, the positive predictive value $93 \%$, the negative predictive value $86 \%$ and the diagnostic accuracy $92 \%$.

The semi-quantitative results of DaTSCAN were analysed with Kruskal-Wallis one-way ANOVA with Mann Whitney U Test as post hoc. There was no significant difference in the striatum uptake ratio between PD (median 2.18, 95\% CI 1.91 and 2.47) and APS (median 2.12, 95\% CI 1.81 and 2.51). However, there was a significant difference ( $\mathrm{p}<0.001,3$ df) between PD and Non-parkinsonism (median 4.79, 95\% CI 4.27 and 5.47) as well as between APS and Non-parkinsonism ( $p<0.001,3 \mathrm{df}$, Figure 3a). The putamen/caudate uptake ratio in PD (median 0.730, 95\% CI 0.714 and 0.748 ) was significantly lower ( $\mathrm{p}=0.006,3 \mathrm{df}$ ) compared to APS (median 0.793, 95\% CI 0.768 and 0.800$)$ and significantly lower $(\mathrm{p}<0.001,3 \mathrm{df})$ than Non-parkinsonism (median 0.819 , 95\% CI 0.807 and 0.847), as shown in Figure 3b.

There was a strong positive correlation (Pearson correlation $r=0.697, p<0.001$ ) between B-SIT and the striatum uptake ratio for the PD group. A weak positive correlation between B-SIT and the striatum uptake ratio was shown even for the APS (Pearson correlation $\mathrm{r}=0.352, \mathrm{p}=0.181$ ) and Non-parkinsonism groups (Pearson correlation $\mathrm{r}=0.408, \mathrm{p}=0.131$ ). There was no correlation between B-SIT and the putamen/caudate ratio for any of the diagnostic groups.

As the majority of APS patients were classified as Grade 1 and the majority of PD patients were classified as Grade 2, we employed binary logistic regression to evaluate if the combination of B-SIT and Grade 1 or Grade 2 can increase the predictive value for PD and APS. Regression analysis for the other grades of visual assessment was not performed due to insufficient amount of data. The correct classification for APS was $43.8 \%$ when solely using B-SIT, $56.3 \%$ when solely using Grade 1 uptake pattern, and $62.5 \%$ when combining these two methods. Similarly, the overall classification for both PD and APS was improved by the combination of BSIT and Grade 1 or Grade 2, as shown in Table 3. 


\section{DISCUSSION}

The main findings of this study illustrate that DaTSCAN has better diagnostic value than olfactory testing in discriminating parkinsonism from Non-parkinsonism. Moreover, DaTSCAN offers prospect of differentiating PD from APS. However, the combination of DaTSCAN and olfactory testing can increase their predictive value.

\section{Olfaction}

According to this study, olfactory testing can successfully distinguish PD patients from Non-parkinsonism, which is in line with previous studies [22-25]. However, olfactory testing fails in distinguishing PD from APS or APS from Non-parkinsonism. Olfactory function can vary in APS from preserved to differentially impaired. It can be significantly impaired in DLB, mildly impaired in MSA, but within normal range in PSP [26, 27], explaining why the olfactory test cannot differentiate APS from PD or Non-parkinsonism. Other studies indicate that testing the olfactory function may be helpful in distinguishing PD from vascular parkinsonism or druginduced parkinsonism [28, 29]. In this study, we could not recruit enough patients to these two subgroups in order to validate this argument.

The B-SIT score had a sensitivity of $80 \%$ for discriminating parkinsonism from Non-parkinsonism and an even higher sensitivity of $92 \%$ for discriminating PD from Non-parkinsonism. However, its specificity was poor $(40 \%)$ in both cases. These results are comparable to Deeb et al. [22], but not in accordance with other studies where sensitivity and specificity were nearly on the same level [25, 30]. A potential explanation for this divergence could depend on cultural biases and odour familiarity. Even though the B-SIT test is a cross-cultural brief smell test, the Swedish population is not familiar with some of the included odours (i.e. wintergreen and lime in this study), resulting in a lower score even for normosmic patients. None of the participants of this study managed to get a perfect score (12 out of 12). In the present study, we chose not to focus on specific odours that may identify PD more effectively. Selective olfactory loss in PD has been controversial; according to Hahner et al. selective hyposmia is not a reliable method to distinguish PD from Non-parkinsonism [31].

Olfactory loss is among the most common non-motor symptoms in PD patients in the 2- to 10-year period prior to motors symptom [32]. Olfactory testing has been used as a supporting diagnostic tool for several years. Our study can confirm the efficacy of olfactory testing in discriminating PD from Non-parkinsonism; however, olfactory testing has a limited efficacy in differentiating the different types of parkinsonism.

\section{DaTSCAN}


Following the five uptake patterns suggested by Kahraman et al., we found that most PD patients had a Grade 2 (egg-shape) uptake reduction, meaning that dopamine depletion in most PD patients is prominent in putamen, while caudate nucleus retains normal or almost normal function. On the other hand, most APS patients showed a Grade 1 (burst striatum) uptake reduction, suggesting more severe dopamine depletion both in putamen and in caudate nucleus in APS. Both Kahraman et al. and Davidsson et al. have previously published similar results to these findings [7, 8]. Compared to the B-SIT test, the visual assessment of DaTSCAN was more efficient in discriminating parkinsonism from Non-parkinsonism in terms of sensitivity (96\%), specificity (80\%) and diagnostic accuracy (92\%). These results contradict Deeb et al., who suggested that a basic smell test is as sensitive as DaTSCAN [22], but are in accordance with Hong et al. who, by comparing the Cross-Cultural Smell Identification Test and dopamine transporter Positron Emission Tomography (PET), proved that the olfactory test alone has little power in detecting dopaminergic depletion [33].

The semi-quantitative analysis of DaTSCAN showed that the striatum ratio is accurate to differentiate PD from Non-parkinsonism, as well as APS from Non-parkinsonism, but it cannot differentiate PD from APS. These results are in accordance with one meta-analysis on the diagnostic accuracy of DaTSCAN [9] and two previously published studies on the same topic $[10,34]$. However, the putamen/caudate ratio is greater in APS than in PD, suggesting a more uniform dopamine depletion both in putamen and in caudate nucleus in APS patients; this result concurs with the previously discussed visual assessment. Two more studies verify that there is more symmetric loss of dopaminergic nerve terminals in MSA [35] and in PSP [36] compared to PD. This finding could depend on higher severity of clinical symptoms in APS patients at the time of the first clinical evaluation.

There was a strong correlation between B-SIT and the striatum ratio in PD patients, indicating that a lower striatum ratio in a PD patient signals a more severely impaired olfaction. The aforementioned studies by Deeb et al [22]. and Hong et al. [33] also found strong correlation between the score of smell identification test and the DaTSCAN uptake in PD patients. Moreover, binary logistic regression analysis showed that the combination of B-SIT and DaTSCAN has better predictive value, with a higher overall rate of correctly classified PD and APS patients, compared to when these two methods are used separately. As shown in Table 3, the combination of Grade 1 uptake pattern and olfactory testing is a significantly better diagnostic model than Grade 1 alone. The addition of olfactory testing in Grade 2 uptake pattern also leads to a higher rate of correctly classified patients, but this improvement is not statistically significant. In conclusion, B-SIT can be a useful supplementary 
diagnostic tool, despite its lower diagnostic accuracy compared to DaTSCAN, especially for Grade 1 uptake pattern, which is dominated by APS patients.

\section{Discordant results}

As seen in Table 4, seven patients had discordant results. Two normosmic PD patients had abnormal DaTSCAN. According to previously published data, $26 \%$ of PD patients had an olfactory test score within the normal range [11]. This finding is in line with the low specificity of smell test in detecting parkinsonism. One APS patient with clearly impaired olfaction had normal DaTSCAN and can, therefore, be classified as Scans Without Evidence of Dopaminergic Deficit (SWEDD). This patient was receiving dopaminergic medication at the time of examination and was diagnosed with MSA after DaTSCAN; hence, the possible interference of dopaminergic medication cannot be eliminated in that case. Also, one normosmic patient with vascular parkinsonism can be classified as SWEDD. A recently published study compared the clinical and imaging characteristics between SWEDD patients and patients with abnormal DaTSCAN during a 22-month follow-up, and concluded that SWEDD patients had minimal evidence of clinical or imaging progression, suggesting that SWEDD patients are unlikely to have parkinsonism [37]. Three patients received a diagnosis different than parkinsonism, despite abnormal DaTSCAN and impaired olfaction, implying incorrect diagnosis. Dopaminergic deficit has been previously documented in some patients with essential tremor, hinting at a possible association between essential tremor and PD [38]. However, olfaction is normal in the vast majority of patients with essential tremor, or in some cases slightly abnormal [39]. As suggested by a multicentre study, a second DaTSCAN two years after clinical observation can reduce diagnostic uncertainty in patients with inconclusive diagnosis [6].

\section{Limitations of the study}

Our cohort is of a prospective nature and therefore no long-term follow-up data is available. In addition, most of the patients included in the study were not drug-naïve to dopaminergic medication, which could potentially affect the results of DaTSCAN SPECT. As with all the studies on the same topic, there is no gold standard for the diagnosis of parkinsonism, apart from a post-mortem examination of the brain. Finally, information about UPDRS and Hoehn and Yahr scale was not documented in the medical journals of all patients since it is not standard practice; analysis of covariance based on this classification was consequently not possible. However, the results of this study are in line with other previously published studies, which underlines the validity of the findings presented here. 


\section{Conclusions}

In conclusion, the semi-quantitative analysis of DaTSCAN SPECT is an accurate diagnostic tool to differentiate parkinsonism from Non-parkinsonism. Additionally, the visual assessment of DaTSCAN together with the uptake ratio between putamen and caudate nucleus can offer valuable aid in differentiating PD from APS. On the other hand, a smell identification test has lower diagnostic value than DaTSCAN in differentiating parkinsonism from Non-parkinsonism. Olfactory testing is efficient in detecting PD, but it cannot successfully detect APS, and its specificity is low. Nonetheless, the combination of olfactory testing and DaTSCAN imaging has a higher predictive value than these two methods separately, especially for Grade 1 uptake pattern. Therefore olfactory tests may be used as a complementary diagnostic tool in parkinsonian syndromes.

\section{Acknowledgements}

This study was supported by grants from Östergötland County Council (ALF) and from the Swedish Parkinson's Foundation. The authors would like to thank Karl Wahlin and Mats Fredrikson from Linköping Academic Research Centre (LARC) for statistical help.

\section{Conflicts of interest}

The authors declare that they have no conflict of interest.

\section{Ethical Standards}

This study was conducted in accordance with the 1964 Declaration of Helsinki and its later amendments, and was approved by the Regional Ethical Review Board in Linköping, Sweden (registration number 2011/415-31). Informed consent was obtained from all individual participants included in the study. 


\section{REFERENCES}

1. Hughes AJ, Daniel SE, Blankson S, Lees AJ (1993) A clinicopathologic study of 100 cases of Parkinson's disease. Arch Neurol 50:140-148

2. Przedborski S (2007) Etiology and Pathogenesis of Parkinson's Disease<br />. In: Jankovic J, Tolosa E (eds) Parkinson's Disease and Movement Disorders, 5th edn. Lippincott Williams \& Wilkins, Philadelphia, USA, pp 77-91

3. Piccini P, Whone A (2004) Functional brain imaging in the differential diagnosis of Parkinson's disease. Lancet Neurol 3:284-290

4. Scherfler C, Schwarz J, Antonini A, Grosset D, Valldeoriola F, Marek K, Oertel W, Tolosa E, Lees AJ, Poewe W (2007) Role of DAT-SPECT in the diagnostic work up of parkinsonism. Mov Disord 22:1229-1238

5. Bajaj N, Hauser RA, Seibyl J, Kupsch A, Plotkin M, Chen C, Grachev ID (2014) Association between Hoehn and Yahr, Mini-Mental State Examination, age, and clinical syndrome predominance and diagnostic effectiveness of ioflupane I 123 injection (DaTSCAN) in subjects with clinically uncertain parkinsonian syndromes. Alzheimers Res Ther 6:67-014-0067-0. eCollection 2014

6. Tolosa E, Borght TV, Moreno E, DaTSCAN Clinically Uncertain Parkinsonian Syndromes Study Group (2007) Accuracy of DaTSCAN (123I-Ioflupane) SPECT in diagnosis of patients with clinically uncertain parkinsonism: 2-year follow-up of an open-label study. Mov Disord 22:2346-2351

7. Davidsson A, Georgiopoulos C, Dizdar N, Granerus G, Zachrisson H (2014) Comparison between visual assessment of dopaminergic degeneration pattern and semi-quantitative ratio calculations in patients with Parkinson's disease and Atypical Parkinsonian syndromes using DaTSCAN((R)) SPECT. Ann Nucl Med 28:851-859

8. Kahraman D, Eggers C, Schicha H, Timmermann L, Schmidt M (2012) Visual assessment of dopaminergic degeneration pattern in 123I-FP-CIT SPECT differentiates patients with atypical parkinsonian syndromes and idiopathic Parkinson's disease. J Neurol 259:251-260

9. Vlaar AM, van Kroonenburgh MJ, Kessels AG, Weber WE (2007) Meta-analysis of the literature on diagnostic accuracy of SPECT in parkinsonian syndromes. BMC Neurol 7:27

10. Vlaar AM, de Nijs T, Kessels AG, Vreeling FW, Winogrodzka A, Mess WH, Tromp SC, van Kroonenburgh MJ, Weber WE (2008) Diagnostic value of 123I-ioflupane and 123I-iodobenzamide SPECT scans in 248 patients with parkinsonian syndromes. Eur Neurol 59:258-266

11. Hawkes CH, Shephard BC, Daniel SE (1997) Olfactory dysfunction in Parkinson's disease. J Neurol Neurosurg Psychiatry 62:436-446

12. Hawkes CH, Shephard BC, Daniel SE (1999) Is Parkinson's disease a primary olfactory disorder?. QJM 92:473-480

13. Doty RL, Shaman P, Dann M (1984) Development of the University of Pennsylvania Smell Identification Test: a standardized microencapsulated test of olfactory function. Physiol Behav 32:489-502

14. Doty RL, Deems DA, Stellar S (1988) Olfactory dysfunction in parkinsonism: a general deficit unrelated to neurologic signs, disease stage, or disease duration. Neurology 38:1237-1244

15. Tissingh G, Berendse HW, Bergmans P, DeWaard R, Drukarch B, Stoof JC, Wolters EC (2001) Loss of olfaction in de novo and treated Parkinson's disease: possible implications for early diagnosis. Mov Disord $16: 41-46$ 
16. Berendse HW, Booij J, Francot CM, Bergmans PL, Hijman R, Stoof JC, Wolters EC (2001) Subclinical dopaminergic dysfunction in asymptomatic Parkinson's disease patients' relatives with a decreased sense of smell. Ann Neurol 50:34-41

17. Wenning GK, Shephard B, Hawkes C, Petruckevitch A, Lees A, Quinn N (1995) Olfactory function in atypical parkinsonian syndromes. Acta Neurol Scand 91:247-250

18. Liberini P, Spano P, Cuello AC (1993) Alzheimer's disease and Lewy body dementia. Br J Psychiatry 163:693-4; author reply 694-5

19. Driver-Dunckley E, Adler CH, Hentz JG, Dugger BN, Shill HA, Caviness JN, Sabbagh MN, Beach TG, Arizona Parkinson Disease Consortium (2014) Olfactory dysfunction in incidental Lewy body disease and Parkinson's disease. Parkinsonism Relat Disord 20:1260-1262

20. Berardelli A, Wenning GK, Antonini A, Berg D, Bloem BR, Bonifati V, Brooks D, Burn DJ, Colosimo C, Fanciulli A, Ferreira J, Gasser T, Grandas F, Kanovsky P, Kostic V, Kulisevsky J, Oertel W, Poewe W, Reese JP, Relja M, Ruzicka E, Schrag A, Seppi K, Taba P, Vidailhet M (2013) EFNS/MDS-ES/ENS [corrected] recommendations for the diagnosis of Parkinson's disease. Eur J Neurol 20:16-34

21. Riklund K, Mo SJ, Larsson A, Collett FJ, Edenbrandt L, Broedsgaard E, Lonsdale MN, Friberg L (2010) Semi-Quantification of DAT SPECT Images - Survey of Normal Reference Limits Used at Different Hospitals. Eur J Nucl Med Mol Imaging 37:S400

22. Deeb J, Shah M, Muhammed N, Gunasekera R, Gannon K, Findley LJ, Hawkes CH (2010) A basic smell test is as sensitive as a dopamine transporter scan: comparison of olfaction, taste and DaTSCAN in the diagnosis of Parkinson's disease. QJM 103:941-952

23. Picillo M, Pellecchia MT, Erro R, Amboni M, Vitale C, Iavarone A, Moccia M, Allocca R, Orefice G, Barone P (2014) The use of University of Pennsylvania Smell Identification Test in the diagnosis of Parkinson's disease in Italy. Neurol Sci 35:379-383

24. Hakyemez HA, Veyseller B, Ozer F, Ozben S, Bayraktar GI, Gurbuz D, Cetin S, Yildirim YS (2013) Relationship of olfactory function with olfactory bulbus volume, disease duration and Unified Parkinson's disease rating scale scores in patients with early stage of idiopathic Parkinson's disease. J Clin Neurosci 20:1469-1470

25. Double KL, Rowe DB, Hayes M, Chan DK, Blackie J, Corbett A, Joffe R, Fung VS, Morris J, Halliday GM (2003) Identifying the pattern of olfactory deficits in Parkinson disease using the brief smell identification test. Arch Neurol 60:545-549

26. Hawkes CH, Doty RL (2009) . In: The Neurology of Olfaction, 1st edn. Cambridge University Press, New York, pp 175-181

27. Hoyles K, Sharma JC (2013) Olfactory loss as a supporting feature in the diagnosis of Parkinson's disease: a pragmatic approach. J Neurol 260:2951-2958

28. Katzenschlager R, Zijlmans J, Evans A, Watt H, Lees AJ (2004) Olfactory function distinguishes vascular parkinsonism from Parkinson's disease. J Neurol Neurosurg Psychiatry 75:1749-1752

29. Brigo F, Erro R, Marangi A, Bhatia K, Tinazzi M (2014) Differentiating drug-induced parkinsonism from Parkinson's disease: an update on non-motor symptoms and investigations. Parkinsonism Relat Disord 20:808814

30. Rodriguez-Violante M, Gonzalez-Latapi P, Camacho-Ordonez A, Martinez-Ramirez D, Morales-Briceno H, Cervantes-Arriaga A (2014) Comparing the accuracy of different smell identification tests in Parkinson's disease: relevance of cultural aspects. Clin Neurol Neurosurg 123:9-14 
31. Hahner A, Maboshe W, Baptista RB, Storch A, Reichmann H, Hummel T (2013) Selective hyposmia in Parkinson's disease?. J Neurol 260:3158-3160

32. Pont-Sunyer C, Hotter A, Gaig C, Seppi K, Compta Y, Katzenschlager R, Mas N, Hofeneder D, Brucke T, Bayes A, Wenzel K, Infante J, Zach H, Pirker W, Posada IJ, Alvarez R, Ispierto L, De Fabregues O, Callen A, Palasi A, Aguilar M, Marti MJ, Valldeoriola F, Salamero M, Poewe W, Tolosa E (2015) The onset of nonmotor symptoms in Parkinson's disease (the ONSET PD study). Mov Disord 30:229-237

33. Hong JY, Chung SJ, Lee JE, Sunwoo MK, Lee PH, Sohn YH (2013) Predictive value of the smell identification test for nigrostriatal dopaminergic depletion in Korean tremor patients. Parkinsonism Relat Disord 19:10181021

34. Mo SJ, Linder J, Forsgren L, Larsson A, Johansson L, Riklund K (2010) Pre- and postsynaptic dopamine SPECT in the early phase of idiopathic parkinsonism: a population-based study. Eur J Nucl Med Mol Imaging $37: 2154-2164$

35. Varrone A, Marek KL, Jennings D, Innis RB, Seibyl JP (2001) (123)I]beta-CIT SPECT imaging demonstrates reduced density of striatal dopamine transporters in Parkinson's disease and multiple system atrophy. Mov Disord 16:1023-1032

36. Antonini A, Benti R, De Notaris R, Tesei S, Zecchinelli A, Sacilotto G, Meucci N, Canesi M, Mariani C, Pezzoli G, Gerundini P (2003) 123I-Ioflupane/SPECT binding to striatal dopamine transporter (DAT) uptake in patients with Parkinson's disease, multiple system atrophy, and progressive supranuclear palsy. Neurol Sci 24:149-150

37. Marek K, Seibyl J, Eberly S, Oakes D, Shoulson I, Lang AE, Hyson C, Jennings D, Parkinson Study Group PRECEPT Investigators (2014) Longitudinal follow-up of SWEDD subjects in the PRECEPT Study. Neurology 82:1791-1797

38. Shahed J, Jankovic J (2007) Exploring the relationship between essential tremor and Parkinson's disease. Parkinsonism Relat Disord 13:67-76

39. Hawkes C (2003) Olfaction in neurodegenerative disorder. Mov Disord 18:364-372 


\section{FIGURES}

Fig.1 Classification of DaTSCAN uptake. The visual assessment of DaTSCAN SPECT was based on these five striatal uptake patterns of DaTSCAN, as previously proposed by Kahraman et al. [8]

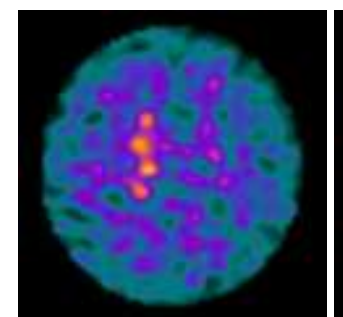

Grade 1

(burst striatum)

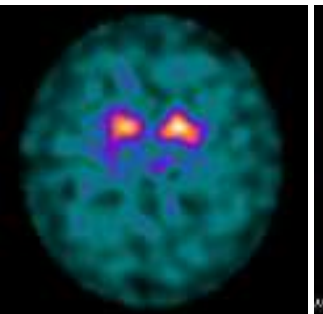

Grade 2

(egg shape)

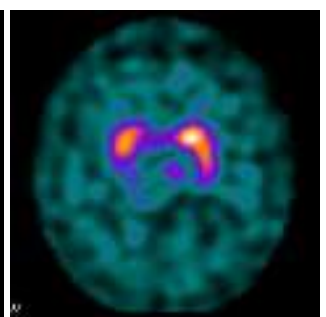

Grade 3

(mixed type)

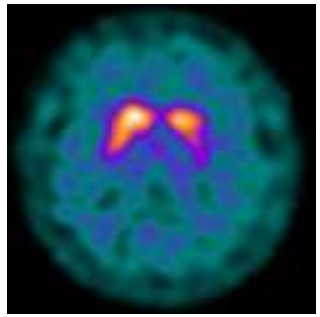

Grade 4 (eagle wing)

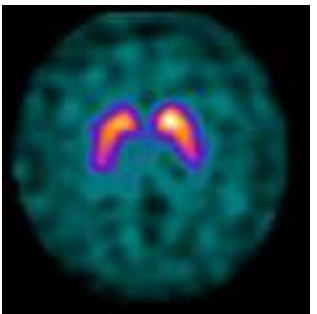

Grade 5 (normal) 
Fig.2 Distribution of PD and APS patients in five striatal uptake grades. All patients were classified in five different uptake patterns (as previously proposed by Kahraman et al.), after visual assessment of DaTSCAN imaging. Significant differences were detected between PD and APS for Grade $1(\mathrm{p}=0.025)$ and Grade $2(\mathrm{p}=0.001)$. PD: Parkinson's disease, APS: Atypical Parkinsonism, * if $\mathrm{p}<0.05, * *$ if $\mathrm{p}<0.01$ [8]

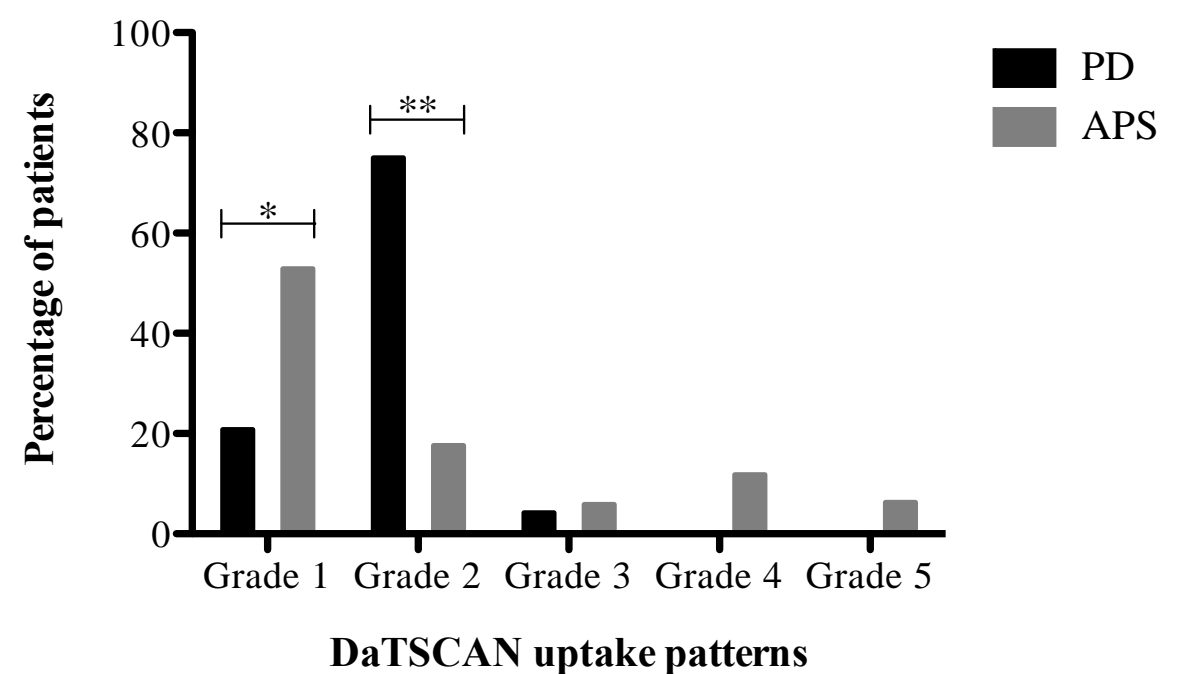


Fig.3 DaTSCAN uptake ratios for PD, APS and Non-parkinsonism. The box plots represent the two different uptake ratios, which were calculated using the 3D EXINI DAT software (EXINI Diagnostics AB, Lund, Sweden). The box horizontal upper and lower limits represent the $75^{\text {th }}$ and $25^{\text {th }}$ percentiles, respectively, and the horizontal line within each box represents the median. a) The striatum ratio was significantly lower $(\mathrm{p}<0.001)$ both for PD and APS compared to Non-parkinsonism. b) The Putamen/Caudate ratio was significantly lower for PD compared to APS ( $\mathrm{p}=0.006)$ and Non-parkinsonism $(\mathrm{p}<0.001)$; there was no significant difference between APS and Non-parkinsonism. PD: Parkinson's disease, APS: Atypical Parkinsonism, Non-p: Non-parkinsonian syndromes, $* *$ if $\mathrm{p}<0.01$

$\mathbf{a}$

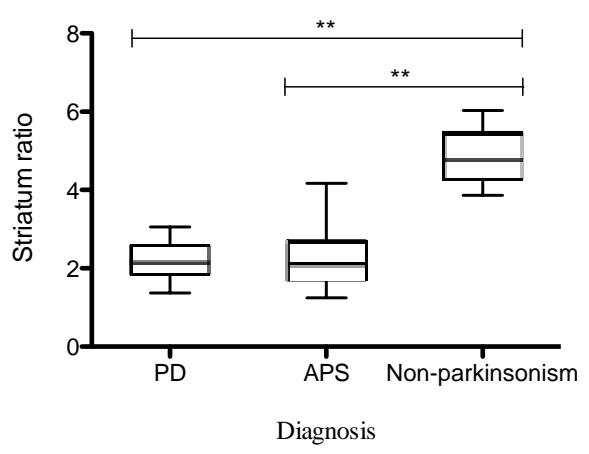

b

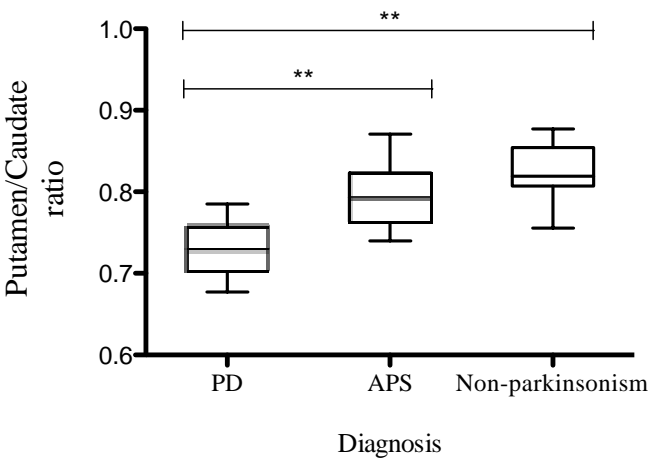




\section{The diagnostic value of dopamine transporter imaging and olfactory testing in patients with parkinsonian syndromes}

\section{Charalampos Georgiopoulos ${ }^{1,6}$}

Anette Davidsson ${ }^{2}$

Maria Engström ${ }^{3,6}$

Elna-Marie Larsson ${ }^{4}$

Helene Zachrisson ${ }^{2, \#}$

Nil Dizdar ${ }^{5, \#}$

${ }^{1}$ Department of Radiology and Department of Medical and Health Sciences, Linköping University, 58185 Linköping, Sweden

${ }^{2}$ Department of Clinical Physiology and Department of Medical and Health Sciences, Linköping University, 58185 Linköping, Sweden

${ }^{3}$ Department of Medical and Health Sciences, Linköping University, 58185 Linköping, Sweden

${ }^{4}$ Department of Surgical Sciences/Radiology, Uppsala University, Akademiska sjukhuset, 75185 Uppsala, Sweden

${ }^{5}$ Department of Neurology and Department of Clinical and Experimental Medicine, Linköping University, 58185 Linköping, Sweden

${ }^{6}$ Center for Medical Image Science and Visualization (CMIV), Linköping University, 58185 Linköping, Sweden

\# Helene Zachrisson and Nil Dizdar have contributed equal parts for last authorship

\section{Corresponding author:}

Charalampos Georgiopoulos

Tel: +46 101032700

Email: Charalampos.Georgiopoulos@regionostergotland.se 


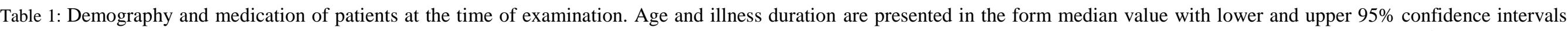

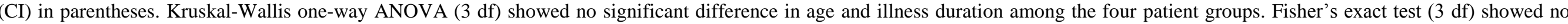

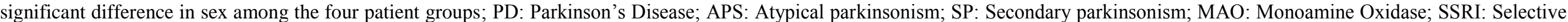
Serotonin Reuptake Inhibitor; NaSSA: Noradrenergic and Specific Serotonergic Antidepressants; SNRI: Serotonin and Noradrenalin Reuptake Inhibitors.

\begin{tabular}{|c|c|c|c|c|c|}
\hline & PD & APS & SP & Non-parkinsonism & $\mathbf{p}$ \\
\hline Number of patients & 24 & 16 & 5 & 15 & \\
\hline Age (years) & 70.5 (95\% CI: 67 and 73) & $65.5(95 \%$ CI: 61 and 72) & 77 (95\% CI: 47 and 80) & 66 (95\% CI: 60 and 71) & 0.257 \\
\hline Sex (male/female) & $13 / 11$ & $9 / 7$ & $5 / 0$ & $7 / 8$ & 0.226 \\
\hline Illness duration (years) & 3.5 (95\% CI: 2 and 4) & 4 (95\% CI: 2 and 6.5) & 4 (95\% CI: 3 and 7) & 6 (95\% CI: 3.5 and 13) & 0.260 \\
\hline Levodopa & 16 & 10 & 0 & 2 & \\
\hline Dopamine Agonist & 7 & 1 & 0 & 1 & \\
\hline MAO inhibitor & 3 & 1 & 0 & 0 & \\
\hline Benzodiazepine & 2 & 2 & 1 & 3 & \\
\hline Antiepileptic & 3 & 2 & 2 & 3 & \\
\hline SSRI & 0 & 1 & 1 & 1 & \\
\hline Tricyclic Antidepressants & 0 & 0 & 0 & 1 & \\
\hline NaSSA & 2 & 1 & 1 & 1 & \\
\hline SNRI & 1 & 2 & 0 & 1 & \\
\hline Serotonin Receptor Agonist & 1 & 0 & 0 & 0 & \\
\hline Antipsychotic & 0 & 1 & 1 & 0 & \\
\hline No medication & 5 & 2 & 1 & 8 & \\
\hline
\end{tabular}




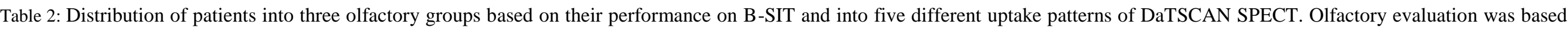

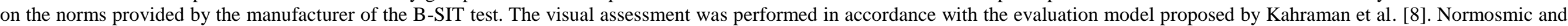

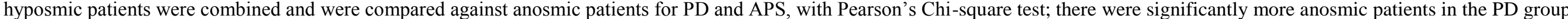

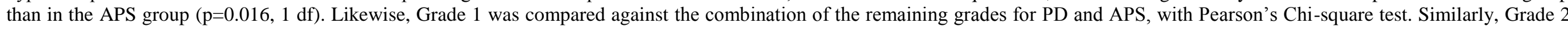

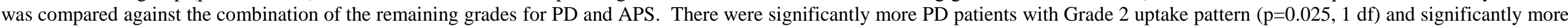

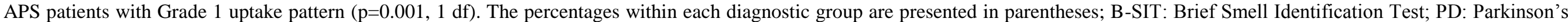
disease; APS: Atypical parkinsonism; SP: Secondary parkinsonism.

\begin{tabular}{|c|c|c|c|c|c|}
\hline & & PD $(n=24)$ & $\operatorname{APS}(n=16)$ & $\mathbf{S P}(n=5)$ & Non-parkinsonism $(n=15)$ \\
\hline \multirow{3}{*}{ B-SIT } & Normosmic & $2(8 \%)$ & $5(31 \%)$ & $2(40 \%)$ & $6(40 \%)$ \\
\hline & Hyposmic & $5(21 \%)$ & $6(38 \%)$ & $1(20 \%)$ & $8(53 \%)$ \\
\hline & Anosmic & $17(71 \%)$ & $5(31 \%)$ & $2(40 \%)$ & $1(7 \%)$ \\
\hline \multirow{5}{*}{$\begin{array}{l}\text { Visual assessment of } \\
\text { DaTSCAN SPECT }\end{array}$} & Grade 1 "Burst Striatum" & $5(21 \%)$ & $9(56 \%)$ & $1(20 \%)$ & 0 \\
\hline & Grade 2 "Egg shape” & $18(75 \%)$ & $3(19 \%)$ & $2(40 \%)$ & 0 \\
\hline & Grade 3 "Mixed type" & $1(4 \%)$ & $1(6 \%)$ & $1(20 \%)$ & $1(7 \%)$ \\
\hline & Grade 4 "Eagle wing" & 0 & $2(13 \%)$ & 0 & $2(13 \%)$ \\
\hline & Grade 5 "Normal" & 0 & $1(6 \%)$ & $1(20 \%)$ & $12(80 \%)$ \\
\hline
\end{tabular}




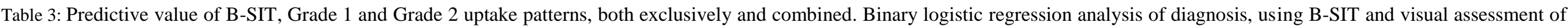

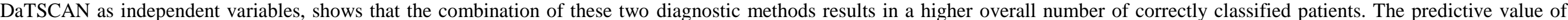

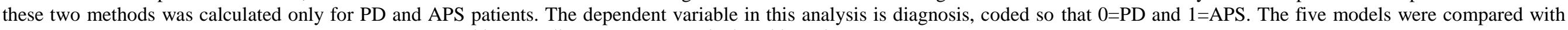

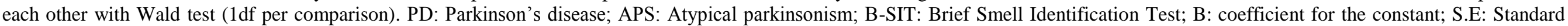
Error of the coefficient for the constant; O.R: Odds Ratio.

\begin{tabular}{|c|c|c|c|c|c|c|c|c|c|c|}
\hline Model & & Model comparison & Log likelihood & Nagelkerke $\mathbf{R}^{2}$ & $\begin{array}{l}\text { Overall correctly } \\
\text { classified }\end{array}$ & & B & S.E. & O.R. & $\mathbf{p}$ \\
\hline 1 & B-SIT & & 47.339 & 0.203 & $70 \%$ & & 0.317 & 0.136 & 1.373 & 0.020 \\
\hline 2 & Grade 1 uptake pattern of DaTSCAN & & 48.539 & 0.168 & $70 \%$ & & 1.586 & 0.712 & 4.967 & 0.026 \\
\hline 3 & Grade 2 uptake pattern of DaTSCAN & & 40.924 & 0.373 & $77.5 \%$ & & -2.565 & 0.795 & 0.077 & 0.001 \\
\hline \multirow[b]{2}{*}{4} & \multirow{2}{*}{$\begin{array}{l}\text { Combination of B-SIT and Grade } 1 \text { uptake } \\
\text { pattern }\end{array}$} & $\mathrm{p}<0.02^{1}$ & \multirow[b]{2}{*}{41.718} & \multirow[b]{2}{*}{0.353} & \multirow[b]{2}{*}{$72.5 \%$} & B-SIT & 0.348 & 0.147 & 1.417 & 0.018 \\
\hline & & $\mathrm{p}<0.001^{2}$ & & & & Grade 1 & 1.800 & 0.800 & 6.048 & 0.025 \\
\hline \multirow{3}{*}{5} & \multirow{3}{*}{$\begin{array}{l}\text { Combination of B-SIT and Grade } 2 \text { uptake } \\
\text { pattern }\end{array}$} & $\mathrm{p}<0.002^{1}$ & \multirow{3}{*}{37.166} & \multirow{3}{*}{0.461} & \multirow{3}{*}{$82.5 \%$} & B-SIT & 0.284 & 0.155 & 1.328 & 0.067 \\
\hline & & & & & & & & & & \\
\hline & & $\mathrm{p}>0.05^{3}$ & & & & Grade 2 & -2.448 & 0.841 & 0.086 & 0.004 \\
\hline
\end{tabular}

${ }^{1}$ Comparison with Model 1 (Wald test)

${ }^{2}$ Comparison with Model 2 (Wald test)

${ }^{3}$ Comparison with Model 3 (Wald test) 
Table 4: Characteristics of patients with discordant test results; B-SIT: Brief Smell Identification Test; PD: Parkinson's disease; APS: Atypical parkinsonism; SP: Secondary parkinsonism.

\begin{tabular}{|c|c|c|c|c|c|c|c|}
\hline Patient & Age & Sex & Diagnosis & Symptom Duration & DaTSCAN & Visual Assessment Grade & B-SIT score/12 \\
\hline P1 & 32 & Male & PD & 2 years & Abnormal & 3 & 11 \\
\hline $\mathrm{P} 2$ & 70 & Female & PD & 2 years & Abnormal & 2 & 9 \\
\hline P3 & 61 & Male & APS & 1 year & Normal & 5 & 5 \\
\hline P4 & 75 & Male & SP & 4 years & Normal & 5 & 10 \\
\hline P5 & 57 & Male & Non-parkinsonism & 9 years & Abnormal & 4 & 8 \\
\hline P6 & 70 & Male & Non-parkinsonism & 2 years & Abnormal & 4 & 8 \\
\hline P7 & 69 & Male & Non-parkinsonism & 24 years & Abnormal & 3 & 6 \\
\hline
\end{tabular}

